\title{
Erratum: Probing the Electronic Structure of the Hemoglobin Active Center in Physiological Solutions [Phys. Rev. Lett. 102, 068103 (2009)]
}

\author{
Emad F. Aziz, Niklas Ottosson, Sébastien Bonhommeau, Nora Bergmann, \\ Wolfgang Eberhardt, and Majed Chergui \\ (Received 19 July 2017; published 10 August 2017)
}

DOI: 10.1103/PhysRevLett.119.069902

We would like to withdraw part of the Letter referenced above.

In view of the recent withdrawal of a paper in Nature Chemistry [1], we reinvestigated the Hemin data published in our Letter in Physical Review Letters as it seems that the same data have been used in both publications. Unfortunately, we found some inconsistencies between the published trace [Fig. 1(b)] in our Letter and some of the traces found in unpublished drafts of the Letter as well as the trace published in Nature Chemistry. This refers to some details of the reported line shape as well as the concentration value of the sample given in either paper (1 mM vs $3 \mathrm{mM})$. The original data is no longer available and therefore we have decided to ask for a withdrawal of this data published in the Letter.

The other data, and especially the data of the $3 \mathrm{mM}$ solution of met-hemoglobin in water, Fig. 1(a), are correct to the best of our knowledge. The discussions and conclusions referring to these data remain valid. The sample description was given in the text but unfortunately some of the information was placed in the Supplemental Material. The supplier specified that the $\mathrm{Hb}$ was exposed to air and therefore $(>98 \%)$ is in the oxidized state referred to as met-hemoglobin.

We also want to correct the expression "physiological solution" used in the title and repeatedly in the text. This may have led to some misunderstandings. It should be replaced by "solution at a concentration close to physiological values" throughout the Letter. The average $\mathrm{Hb}$ concentration in human blood ranges from $1.9 \mathrm{mM}$ to $2.7 \mathrm{mM}[2,3]$.

[1] E. F. Aziz, H. Rittmann-Frank, K. Lange, S. Bonhommeau, and M. Chergui, Nat. Chem. 2, 853 (2010).

[2] R. Hardison, J. Exp. Biol. 201, 1099 (1998).

[3] M. Häggström, Wikiversity J. Med. 1, 8 (2014). 